\title{
Prognostic relevance of remission and measurable residual disease status in AML patients prior to reduced intensity or non-myeloablative allogeneic stem cell transplantation
}

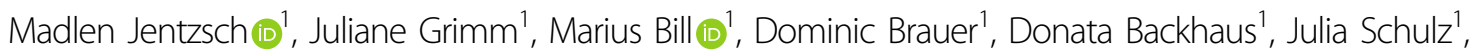 \\ Karoline Goldmann ${ }^{1}$, Dietger Niederwieser [1]', Uwe Platzbecker ${ }^{1}$ and Sebastian Schwind (10)
}

Dear Editor,

Acute myeloid leukemia (AML) presents highly heterogeneous, calling for individualized treatment approaches. Allogeneic hematopoietic stem cell transplantation (HSCT) offers the consolidation treatment with the highest chance of sustained remission for most AML patients ${ }^{1}$. In patients refractory to induction therapy or suffering early relapse, allogeneic HSCT may be performed as a salvage therapy despite the detection of active disease ${ }^{2}$.

At AML diagnosis the European LeukemiaNet (ELN) 2017 risk stratification identifies three prognostically relevant groups, also in patients receiving allogeneic HSCT for consolidation ${ }^{1,3}$. Measurable residual disease (MRD) evaluation at various time points during the disease course, including prior to HSCT, has been shown to provide valuable additional risk stratification in AML patients independently of the applied MRD marker and method $^{4-9}$. Importantly, in an AML cohort receiving allogeneic HSCT, one study showed that outcomes of MRD-positive (MRD ${ }^{\text {pos }}$ ) patients were similarly dismal as in patients transplanted with morphologic active disease ${ }^{10}$. However, the median age in this analysis was 50 years, and all patients received myeloablative (MAC) conditioning, leaving open questions regarding individuals not eligible for intensive conditioning therapies. Here, reduced intensity (RIC) or non-myeloablative (NMA) conditioning is

\footnotetext{
Correspondence: Sebastian Schwind (Sebastian.Schwind@medizin.uni-leipzig.de) 'Medical Clinic and Policlinic 1, Hematology, Cellular Therapy and Hemostaseology, Leipzig University Hospital, Leipzig, Germany Presented in part at the 2020 Annual Meeting of the American Society of Hematology in San Diego/USA and published in abstract form.
}

being applied where disease control increasingly relies on immunological graft-versus-leukemia $(\mathrm{GvL})$ effects ${ }^{11}$.

To compare the prognostic significance of the presence of a morphologic remission and the MRD status prior to performing RIC- or NMA-HSCT, as well as the impact of GvL effects, we retrospectively analyzed 392 AML patients who received an allogeneic HSCT. All patients received NMA- (74\%) or RIC-HSCT (26\%) at a median age of 63.1 (range 21.4-76.8) years with either active disease (33\%) or in morphologic complete remission (CR) or $\mathrm{CR}$ with incomplete peripheral recovery (CRi, 67\%). Details on the applied conditioning regimens are given in the Supplementary Information. Patients' characteristics are shown in Supplementary Table S1. Median follow-up after HSCT was 2.8 years. Written informed consent was obtained from all patients in accordance with the Declaration of Helsinki. MRD status at HSCT was evaluated for all patients transplanted in CR/CRi using quantitative polymerase chain reaction for at least one of the targets NPM1 mutation, BAALC/ABL1, MN1/ABL1, or $W T 1 / A B L 1$ expression adapting the previously published cut-offs ${ }^{5-7}$. Patients with at least one positive test result were regarded as MRD ${ }^{\text {pos }}$.

After NMA- or RIC-HSCT patients transplanted in MRD $^{\text {neg }}$ remission had the best outcomes which also remained significant in multivariate analyses (Supplementary Table S2). With respect to the cumulative incidence of relapse (CIR) rates, our data resembled those following MAC-HSCT ${ }^{10}$, with equally high CIR rates around $50-60 \%$ in patients transplanted with $\mathrm{MRD}^{\text {pos }}$ or active disease (Supplementary Fig. S1A). These resultsdespite different MRD assessment methods (molecular vs 

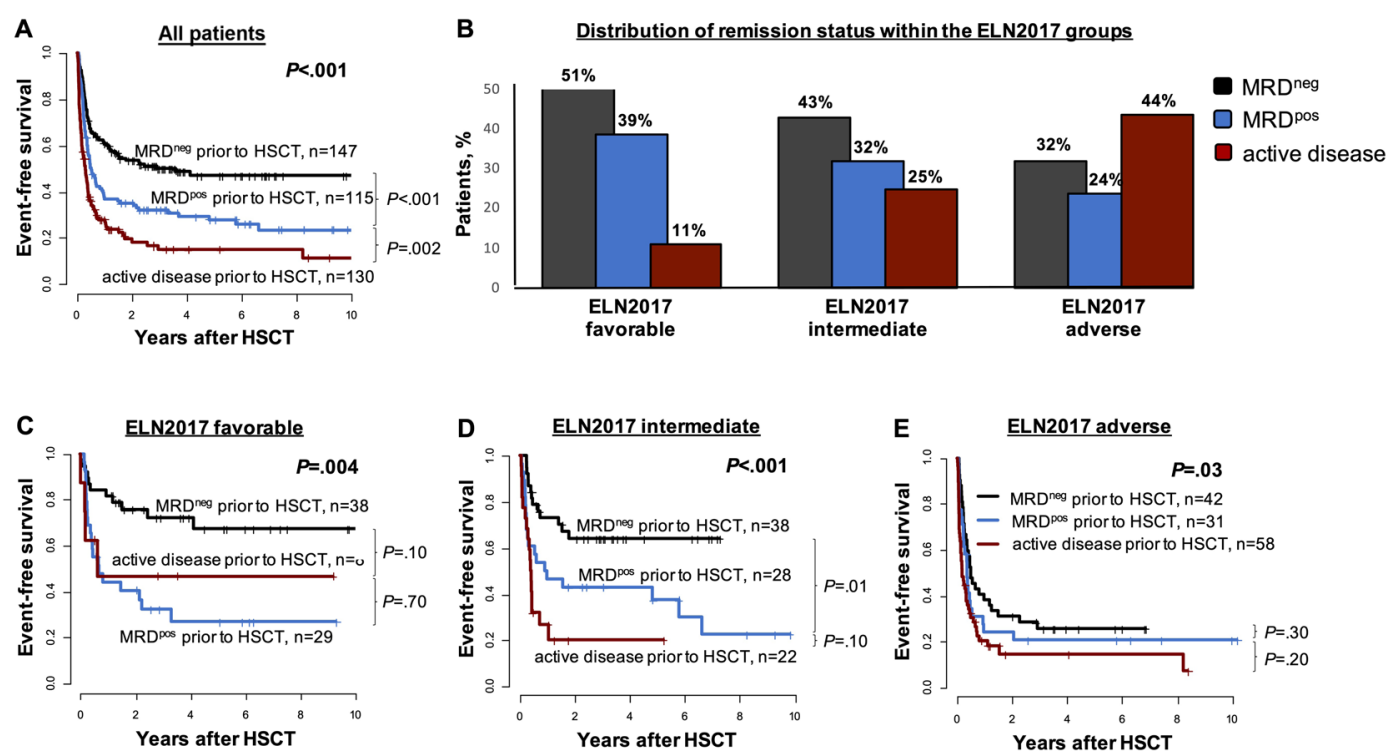

Fig. 1 Outcome and ELN2017 risk distribution according to remission status prior to allogeneic RIC- or NMA-HSCT (MRD ${ }^{\text {neg }}$ vs MRD ${ }^{\text {pos }}$ vs active disease, $\boldsymbol{n}=\mathbf{3 9 2}$ ). A Event-free survival of the whole patient cohort, $\mathbf{B}$ distribution of the remission status prior to allogeneic RIC- or NMAHSCT according to the ELN2017 risk stratification system, C event-free survival in ELN2017 favorable-risk patients $(n=75)$, D event-free survival in ELN2017 intermediate-risk patients $(n=88)$, and $\mathbf{E}$ event-free survival in ELN2017 adverse risk patients $(n=131)$.

flow-based)-resemble those of Araki et al. However, while non-relapse mortality (NRM) was comparable, overall survival (OS) and event-free survival (EFS) were significantly longer in MRD ${ }^{\text {pos }}$ patients compared to those transplanted with active disease (Supplementary Fig. S1B, S1C and Fig. 1A). This indicates that some MRD ${ }^{\text {pos }}$ patients may be salvaged following relapse after HSCT and may achieve long-term survival even when transplanted in a suboptimal remission state.

In our study, the applied conditioning regimens differed significantly between patients with $\mathrm{MRD}^{\text {neg }}, \mathrm{MRD}^{\text {pos }}$, and active disease prior to HSCT, mostly because FLAMSA RIC-HSCT was purposely administered to individuals with the highest relapse risk, usually identified by an active disease prior to allogeneic HSCT. Consequently, we performed a subanalysis that included only individuals receiving the conditioning with the lowest intensity and without thymoglobulin (NMA, 74\% of our cohort) where we yielded similar results as in the whole patient cohort (Supplementary Fig. S2). These data and the observed comparable outcomes between RIC and NMA conditioned patients transplanted with active disease (Supplementary Fig. S3) indicate that our results are rather independent of the applied conditioning regimen. Thus, the deepest possible remission is necessary prior to HSCT to improve outcomes and should be evaluated as part of individualized prognostication and treatment decisions.

We also separately analyzed the three ELN2017 risk groups. The distribution of the patients within the ELN2017 groups reflected the phenotype aggressiveness and the associated difficulties achieving a deep remission prior to HSCT. While there was a stepwise decrease of MRD ${ }^{\text {neg }}$ and $\mathrm{MRD}^{\mathrm{pos}}$ patients, the number of patients transplanted with the active disease increased from ELN2017 favorable to intermediate to adverse risk (Fig. 1B). We observed the strongest outcome separation between $\mathrm{MRD}^{\text {neg }}, \mathrm{MRD}^{\text {pos }}$, and active disease at HSCT in patients with favorable and intermediate ELN2017 risk AML (Fig. 1C-E and Supplementary Fig. S4): $M_{R}{ }^{\text {neg }}$ patients had significantly longer EFS in favorable $(P=0.004)$ and intermediate $(P<0.001)$. ELN2017 risk compared to those with $\mathrm{MRD}^{\mathrm{pos}}$ or active disease. In ELN2017 adverse risk patients, MRD $^{\text {neg }}$ patients also had improved EFS compared to those with MRD ${ }^{\text {pos }}$ or active disease $(P=0.03)$, but EFS in general was short and the impact of the remission status at HSCT limited.

Intriguingly, a variety of parameters at diagnosis known to associate with worse outcomes in AML patients were already significantly different between patients transplanted with active disease compared to those in $M_{R} D^{\text {neg }}$ or $\mathrm{MRD}^{\text {pos }}$ remission. A higher incidence of secondary AML $(P=0.04)$, a higher genetic risk, including an abnormal $(P<0.001)$, monosomal $(P<0.001)$, or complex karyotype $(P=0.006)$, adverse ELN2017 genetic risk $(P<$ $0.001)$, the presence of TP53 mutations $(P=0.05)$, as well as the absence of NPM1 mutations $(P<0.001)$ were more frequently found in patients transplanted with active disease (Supplementary Table S1). In contrast, $\mathrm{MRD}^{\text {neg }}$ and $M R D^{\text {pos }}$ patients only differed regarding their white blood count and SRSF2 mutation status, which again underlines the importance of a dynamic risk stratification 
during remission. Thus, MRD assessment is especially important in lower or intermediate-risk AML patients, which of course is also true regarding potential consolidation decisions towards allogeneic HSCT.

Since RIC and NMA conditioning regimens rely on GvL effects for disease control we performed a landmark analysis of patients surviving longer than 100 days after HSCT to evaluate the prognostic impact of the presence of chronic graft versus host disease (GvHD) as a known surrogate marker for GvL effects. In the entire set the presence of chronic GvHD favorably impacted outcomes following RIC- or NMA-HSCT in univariate and multivariate analyses (Supplementary Table S3). We observed longer OS and EFS for MRD ${ }^{\text {pos }}$ patients compared to patients transplanted with active disease, as well as a favorable effect of chronic GvHD in patients with $M R D^{\text {neg }}$, by trend in patients with $M R D^{\text {pos }}$, but no effect in patients transplanted with active disease (Fig. 2 and Supplementary Fig. S5). The GvL impact was reduced in $M R D^{\text {pos }}$ and more or less lost in the group of patients transplanted with active disease. Thus, the strengths of the GvL effect seem to depend on the disease burden at HSCT and may unfold its full potential only in patients with a low disease burden (i.e., $\mathrm{MRD}^{\text {neg }}$ ) to help control AML following HSCT. This observation may also contribute to the outcome differences observed for the different remission status following HSCT.

Today some clinical trials have addressed the remission depth before HSCT in AML. Application of additional therapies in AML patients not in remission at HSCT may introduce deeper responses and longer survival, which is currently evaluated in a prospective clinical trial (ETAL3-ASAP, NCT02461537). Also, the choice of the conditioning regimen and immunosuppression might be helpful in improving outcomes. $M_{R D}{ }^{\text {pos }}$ patients may benefit from more intensive conditioning regimen ${ }^{12}$, application of donor lymphocytes (Supplementary Fig. S6), or the absence of T-cell depletion (Supplementary Fig. S7) ${ }^{13}$ which of course has to be carefully weighed against a potentially higher NRM. Also, donor selection may contribute to improved outcomes of MRD ${ }^{\text {pos }}$ patients, as in previous studies the use of haploidentical donors resulted in better disease control, longer survival, and similar NRM than sibling HSCT in patients transplanted with active disease ${ }^{14}$ or MRD $^{\text {pos }}$ remission ${ }^{15}$.

With respect to the ELN2017 adverse group the observed outcomes, irrespective of the morphologic remission or MRD status are sobering. The very abysmal outcomes of ELN2017 adverse risk patients following
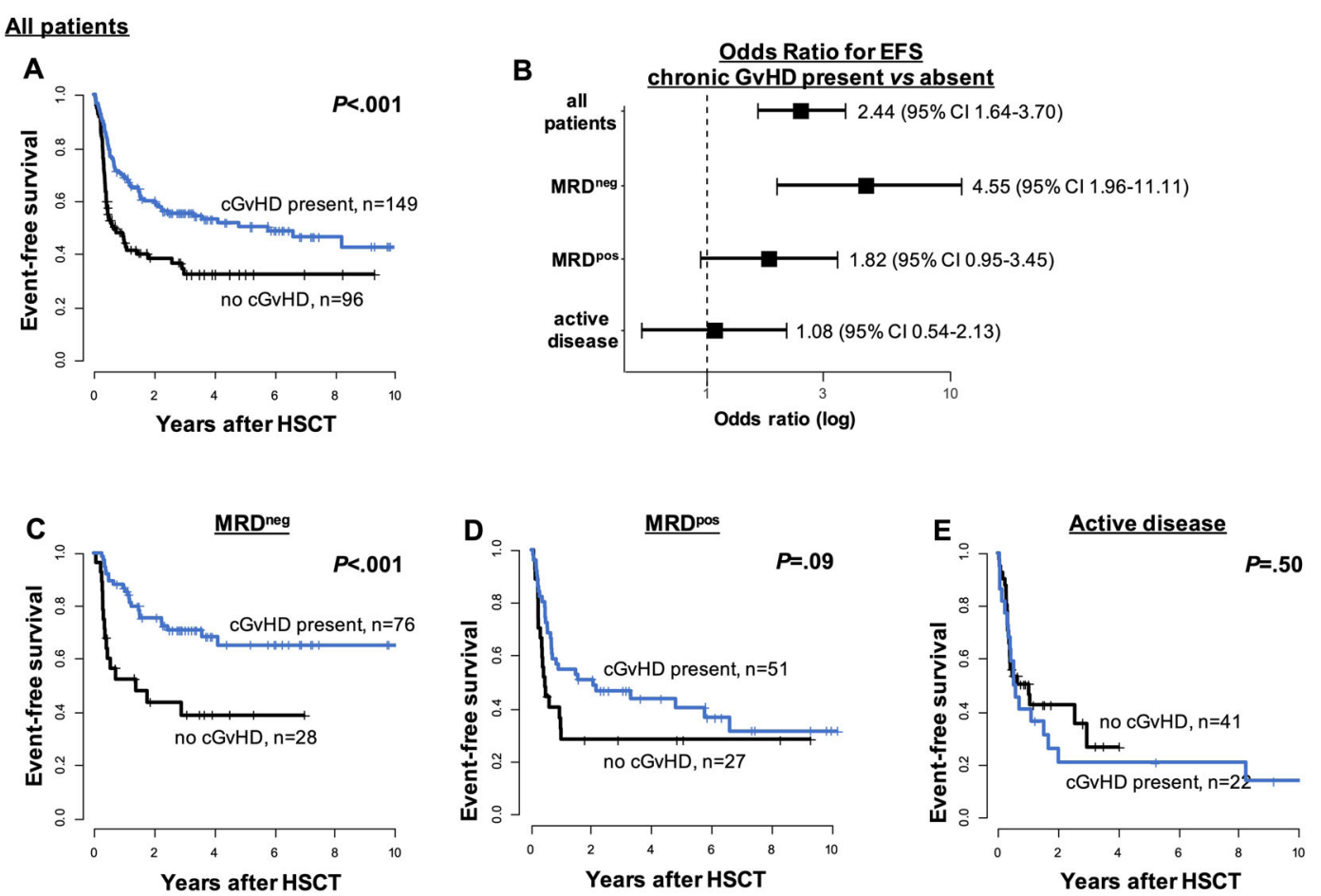

Fig. 2 Event-free survival according to the presence of a chronic GvHD in patients surviving longer than 100 days after allogeneic RIC- or NMA-HSCT (landmark analysis). A All patients $(n=245)$, B forest-plot for the odds-ratio for EFS when chronic GVHD is present for all patients and for the subgroups according to the remission status, $\mathbf{C}$ EFS according to the presence of chronic GvHD in MRD ${ }^{\text {neg }}$ patients $(n=104)$, D MRD ${ }^{\text {pos }}$ patients $(n=78)$, and $\mathbf{E}$ patients with active disease $(n=63)$ at HSCT. 
HSCT call for novel treatment approaches and these patients should be entered into clinical trials whenever possible. Some hope also relies on novel drug combinations that may induce a deeper remission before HSCT and/or approaches regarding maintenance after HSCT.

In their paper, Araki et al. also raised the question of routine use of refined remission criteria to include more sensitive methods, such as the "complete remission without MRD" ${ }^{\prime 9}$. With the here presented data we second this suggestion. Certainly, clinical trials prospectively testing risk-adapted treatment algorithms are needed to change and individualize routine clinical approaches.

In conclusion, our study is the first to indicate comparable high relapse rates in $M R D^{\text {pos }}$ patients and patients receiving RIC- or NMA-HSCT with active disease. MRD $^{\text {neg }}$ patients at HSCT had the best outcomes, an effect that is most pronounced in the ELN2017 favorable and intermediated risk groups. Following RIC or NMA conditioning the GvL effect seems to have the highest impact in patients with a low disease burden at HSCT. The morphologic remission and MRD status at HSCT are prognostically very important in AML patients receiving RIC- or NMA-HSCT and should routinely be assessed to improve individualized prognostication.

\section{Acknowledgements}

This study was supported by the Deutsche Gesellschaft für Innere Medizin (Clinician Scientist Program, M.J.), the Verein Zusammen gegen den Krebs e.V. [S.S.], and the Deutsche Jose Carreras Leukämie-Stiftung (04 R/2016 [S.S.] and PS15/05 [J.G.]). The authors thank Christel Müller, Daniela Bretschneider, Evelin Hennig, Sabine Leiblein, Martina Pleß, Ulrike Bergmann, Janet Bogardt, Annette Jilo, and Dagmar Cron for their help in determining cytogenetic, morphologic, and immunological analyses, and Christine Günther, Scarlett Schwabe, Ines Kovacs, and Kathrin Wildenberger for their help in sample processing.

\section{Author contributions}

M.J. and S.S. contributed to the design and analysis of this study and the writing of the manuscript, and all authors agreed on the final version. M.J., J.G., M.B., D. Brauer, D. Backhaus, J.S., and K.G. carried out the laboratory-based research; M.J. and S.S. performed statistical analyses; and U.P., D.N., and S.S. provided administrative support.

\section{Conflict of interest}

The authors declare no competing interests.

\section{Publisher's note}

Springer Nature remains neutral with regard to jurisdictional claims in published maps and institutional affiliations.
Supplementary information The online version contains supplementary material available at https://doi.org/10.1038/s41408-021-00471-X.

Received: 16 February 2021 Revised: 9 March 2021 Accepted: 25 March 2021

Published online: 29 April 2021

\section{References}

1. Döhner, H. et al. Diagnosis and management of AML in adults: 2017 ELN recommendations from an international expert panel. Blood 129, 424-447 (2017).

2. Schmid, C., Schleuning, M., Ledderose, G., Tischer, J. \& Kolb, H. J. Sequential regimen of chemotherapy, reduced-intensity conditioning for allogeneic stem-cell transplantation, and prophylactic donor lymphocyte transfusion in high-risk acute myeloid leukemia and myelodysplastic syndrome. J. Clin. Oncol. 23, 5675-5687 (2005).

3. Grimm, J. et al. Prognostic impact of the ELN2017 risk classification in patients with AML receiving allogeneic transplantation. Blood Adv. 4, 3864-3874 (2020).

4. Thol, F. et al. Measurable residual disease monitoring by NGS before allogeneic hematopoietic cell transplantation in AML. Blood 132, 1703-1713 (2018).

5. Bill, M. et al. Digital droplet PCR-based absolute quantification of pretransplant NPM1 mutation burden predicts relapse in acute myeloid leukemia patients. Ann. Hematol 97, 1757-1765 (2018).

6. Jentzsch, M. et al. Prognostic impact of blood MN1 copy numbers before allogeneic stem cell transplantation in patients with acute myeloid leukemia. HemaSphere 3, e167 (2019).

7. Jentzsch, M. et al. High BAALC copy numbers in peripheral blood prior to allogeneic transplantation predict early relapse in acute myeloid leukemia patients. Oncotarget 8, 87944-87954 (2017).

8. Ivey, A. et al. Assessment of minimal residual disease in standard-risk AML. N. Engl. J. Med. 374, 422-433 (2016).

9. Schuurhuis, G. J. et al. Minimal/measurable residual disease in AML: a consensus document from the European LeukemiaNet MRD Working Party. Blood 131, 1275-1291 (2018).

10. Araki, D. et al. Allogeneic hematopoietic cell transplantation for acute myeloid leukemia: time to move toward a minimal residual disease-based definition of complete remission? J. Clin. Oncol. 34, 329-336 (2016).

11. Giralt, S. et al. Engraftment of allogeneic hematopoietic progenitor cells with purine analog-containing chemotherapy: harnessing graftversus-leukemia without myeloablative therapy. Blood 89, 4531-4536 (1997).

12. Hourigan, C. S. et al. Impact of conditioning intensity of allogeneic transplantation for acute myeloid leukemia with genomic evidence of residual disease. J. Clin. Oncol. 38, 1273-1283 (2020).

13. Dillon, R. et al. Molecular MRD status and outcome after transplantation in NPM1-mutated AML. Blood 135, 680-688 (2020).

14. Lu, Y. et al. Unmanipulated haplo-identical donor transplantation compared with identical sibling donor had better anti-leukemia effect for refractory/ relapsed acute myeloid leukemia not in remission status. Ann. Hematol. 99, 2911-2925 (2020).

15. Chang, Y. J. et al. Haploidentical allograft is superior to matched sibling donor allograft in eradicating pre-transplantation minimal residual disease of AML patients as determined by multiparameter flow cytometry: a retrospective and prospective analysis. J. Hematol. Oncol. 10, 1-13 (2017). 